\title{
The case for a new system for oversight of research on human subjects
}

Konrad Jamrozik University of Western Australia, Western Australia

\begin{abstract}
The increasing emphasis on evidence-based clinical practice has thrown into sharp focus multiple

deficiencies in current systems of ethical review. This paper argues that a complete overhaul of systems for ethical oversight of studies involving human subjects is now required as developments in medical, epidemiological and genetic research have outstripped existing structures for ethical supervision. It shows that many problems are now evident and concludes that sequential and piecemeal amendments to present arrangements are inadequate to address these. At their core present systems of ethical review still rely on the integrity and judgment of individual investigators. One possible alternative is to train and license research investigators, make explicit their responsibilities and have ethics committees devote much more of their time to monitoring research activity in order to detect those infringing the rules.

(Fournal of Medical Ethics 2000;26:334-339)
\end{abstract}

Keywords: Medical ethics; epidemiology; regulation; genetics

\section{Introduction}

This paper argues that a complete overhaul of systems for ethical oversight of studies involving human subjects is now required. Along with developments in genetic research, the increasing emphasis on evidence-based clinical practice, the child of the marriage between epidemiology and medical research, has thrown into sharp focus many deficiencies in current systems of ethical review. It seems equally clear that sequential and piecemeal amendments to present arrangements are inadequate to address the many problems that are now evident. Instead, the shortcomings of current systems are now so significant and so numerous that a fundamental redesign appears to be a better option than continually amending and adding to existing sets of rules and procedures. One possible alternative is to train and license research investigators, make explicit their responsibilities and have ethics committees devote much more of their time to monitoring research activity in order to detect those infringing the rules. The design and operation of this new model would be equivalent to well established methods of regulating public transport.

\section{Public transport versus medical, epidemiological and genetic research} There are many analogies between the ethical issues surrounding medical, epidemiological and genetic research on human subjects and the expectations of the community with regard to using public transport. These analogies begin with the fact that each activity is associated with some risk, but that the participant cedes responsibility for the conduct of the activity to someone else. In a broad sense passengers on buses and participants in research have willingly compromised their autonomy. Therefore, when we use a bus, for example, we want to be confident that it is designed and maintained well, that the driver has been trained and accredited to operate it, that the journey will be smooth and safe, and that there are checks in the system to maximise the probability that this occurs. Similarly, individuals who are asked to participate in a research project want to be able to assume that it is well designed and well executed, that the investigator is competent to undertake the study, that the study will be run efficiently and safely, and that deviations from good practice will be detected and corrected. Like passengers on a bus, participants in a research study would also like to be able to terminate their journey prematurely, should they desire to do so.

These considerations have led most countries to require that drivers of public buses obtain a special licence and that the buses themselves are subject to special rules of design such as the provision of emergency exits. Behaviour on board buses is also regulated in the interests of public safety. Like all other drivers on the road, drivers of buses must observe stop signs, speed limits and laws regarding drinking and driving. Infringements are liable to detection by red light cameras, radar guns and breathalyser units.

In contrast to rules governing bus drivers, many jurisdictions do not require persons proposing to undertake medical, epidemiological or genetic research to have any particular qualification or to provide any evidence of their competence to carry out such studies. Even more curiously, some countries do license individuals undertaking research involving animals but have no equivalent system for those performing research involving humans. ${ }^{1}$ In much of the English-speaking world, at least, institutional ethics committees (IECs) or institutional review boards do spend enormous amounts of time examining research protocols - the design of the 
bus and its seats-but either their guidelines do not require them to review the logbooks or ask the passengers whether they had a comfortable ride once the study has been completed, ${ }^{2}$ or they do not have time to do so. ${ }^{3}$ It follows that they therefore rely on the investigators' annual reports to assure them that no problems arose. This is equivalent to regulating traffic by asking drivers to report retrospectively each year whether they obeyed every stop sign and speed limit and never drove under the influence of alcohol.

The growing view that all clinical practice should be based on sound evidence reveals that many current systems of ethical review are simply not equipped to cope with modern patterns of clinical research. Two cardinal features of evidence-based practice are the demand for clinical management to be based upon well designed randomised controlled trials wherever possible, and for systematic audit of the impact of such trials on day-to-day practice. ${ }^{4}$ When trials are concerned with detecting moderate benefits, benefits that are nevertheless important if the problem is common or expensive, the studies must be large to have adequate statistical power. ${ }^{5}$ Obtaining unambiguous answers within reasonable amounts of time requires that large studies involve multiple institutions. Systems to oversee research that are based on institutional ethics committees struggle to cope with such projects. Gaining the necessary approvals becomes timeconsuming and expensive, and when the same protocol is submitted to many different IECs, each is tempted to tinker with it. Imagine trying to run the European rail system if each country had different specifications for the sizes of tracks, trains and tunnels.

An intervention study having provided evidence that a particular clinical strategy is to be preferred, it is equally important to monitor subsequent changes in practice and to confirm that the expected benefits are seen. Such observational studies frequently require no contact with individual patients but might provide significantly erroneous results if large numbers or selected subsets of patients are omitted from the review. Many IECs struggle in making the distinction between projects of this kind, where the only hazard is to confidentiality, and true experiments in which the investigator manipulates the way that patients are managed and in which there may be an added risk of physical harm.

\section{The origin and some consequences of the present difficulties}

How can it have happened that many systems of ethical review have failed to keep up with developments in human research and in health service decision making? Part of the answer is that "modern" medical research has a very short history and has displayed meteoric development. Leaving aside foxglove and quinine, the first specific pharmacological treatment for a human disease was Ehrlich's salvarsan in $1907^{6}$ and insulin was first used clinically in $1922 .{ }^{7}$
Undoubtedly the "experiments" undertaken at Dachau and Auschwitz were a major stimulus to the development of current systems of ethical review, leading directly to the Nuremberg Code in $1947^{8}$ and the Declaration of Helsinki in $1964 .^{9}$ They also explain the principal focus of most ethics committees as being the protection of the wellbeing and the rights of the individual participant in research and their "intellectual set" based on intervention studies. Where entirely new treatments, such as insulin and penicillin, have striking effects, sufficient patients to demonstrate them may be found in single hospitals and systems of ethical review based on single institutions are adequate to regulate the research. But, while insulin and penicillin entered day-to-day practice on the basis of what we would now term phase II studies, small case series involving patients in whom all other known approaches to treatment had been tried and had failed, the demands of modern, phase III, randomised controlled trials are fundamentally different. As the first such study, the Medical Research Council trial of streptomycin, ${ }^{10}$ post-dated the second world war, it is not very surprising that codes of ethical practice developed in response to the Nazi "experiments" are now found wanting in terms of the very large, multicentre intervention studies that are common today. One doubts very much that the authors of the Declaration of Helsinki would have thought of the need to accommodate the ISIS-4 trial, which involved 58,050 patients in 1,086 hospitals in 31 countries. ${ }^{11}$

\section{Outflanked}

Institutional ethics committees have also been outflanked by developments in epidemiology. First, being institutional in their focus, they often have little insight into the needs of epidemiology to apply uniform methods to the study of every case of a particular phenomenon as it occurs in a whole population. Here, uniformity is about minimising systematic error and complete coverage concerns avoidance of selection bias, maximising statistical power and minimising random error. Acknowledging their science as being an inexact one, epidemiologists often invest considerable effort in developing, testing and carefully and uniformly applying methods of measurement that control systematic errors as far as is practically possible. When the same protocol is submitted to a series of IECs covering a single population and each committee proposes different changes to it, considerable time can be wasted negotiating a consensus. ${ }^{12}$ Otherwise, avoidable variation is introduced into the study and the likelihood of erroneous (or uninterpretable) results is increased. In parallel fashion, when an IEC refuses to allow recruitment of relevant cases from the institution in which it is based, or insists, for example, on methods of consent that act to dissuade participation in the investigation by relevant individuals, selection bias may be introduced and statistical power is certainly decreased. An example here is a committee that insisted that the investigators in a study of injuries to child pedestrians advise 
the parents of children that they should seek legal advice before completing a questionnaire about how much time their child spent in road environments and under what supervision. ${ }^{13}$

The second general problem is that IECs working within a framework that is primarily concerned with intervention studies on patients are not necessarily in tune with observational epidemiology based on free-living populations of initially healthy individuals. Again, this is probably an accident of history, the Framingham Study having undertaken its baseline surveys in $1948^{14}$ and the first of the adequately designed observational studies of the relationship between smoking and lung cancer, those of Wynder and Graham ${ }^{15}$ and of Doll and Hill, ${ }^{16}$ having been published in 1950 . In these investigations, living participants gave consent to their involvement, but problems arise in large, long term prospective studies when endpoints of interest, particularly fatal ones, involve institutions that are far removed in space and time from the original process of recruitment and consent. While many IECs will allow pertinent clinical information to be divulged to the study team, the most conservative will at least require a full formal application for ethical clearance to be submitted and approved before access to the relevant medical records is granted. ${ }^{17} \mathrm{~A}$ larger proportion is likely to express unease when, as part of the cycle of evidence-based practice, investigators seek to collect details of clinical management and to relate these to outcomes without obtaining consent from the patients at all, the primary question under study being not how individual patients have been treated nor how single practitioners or institutions have performed, but the extent to which a new and proven clinical strategy has been taken up across a whole population and with what impact on that population's health. ${ }^{4}$

As has been discussed elsewhere,${ }^{12}$ a system of ethical review based in single institutions and operating within a framework concerned primarily with the rights and wellbeing of individuals participating in intervention studies threatens to impede the identification of hazards to the public health and of sources of waste, inefficiency and even danger within the health system. But the underlying philosophical stance can also have wider and even more profound implications. When the rights of individuals to privacy are regarded as paramount and given statutory force, as happens, for example, when very restrictive laws on data protection are enacted, certain types of enquiry into the quality of medical care or environmental or occupational hazards become impossible ${ }^{18}$ and the public's desire for confidence that the health system is effective and efficient, and that hospitals, workplaces and other environments are safe is sacrificed on the altar of the confidentiality of individuals.

\section{Outstanding issues}

There are a number of other general areas of ethical regulation with which most existing systems of ethical oversight are ill-equipped or un-eqipped to deal. Some of these are as old as systematic medical research itself, others have only come into focus as medical science has advanced, and yet others appear to be issues that have not been recognised as requiring clear-minded ethical enquiry. The large number and varied nature of these shortcomings add weight to the argument that we have reached the point where fundamental changes in current systems of ethical regulation are required.

\section{MONITORING OF THE CONDUCT OF RESEARCH}

There are numerous reports in the literature describing the problems encountered by epidemiological investigators in obtaining approval for population-wide studies from multiple IECs existing within a single community. ${ }^{12}{ }^{19-22}$ By contrast, the literature is almost silent about the attention that is given to ensuring that, once approved, individual studies are actually conducted in an ethical fashion. To be sure, external audits of records held by individual institutions participating in multicentre trials of new drugs may go some way to correcting this deficiency, but such audits are rarely overseen by the relevant IECs and will not necessarily cover issues such as maintenance of confidentiality even if they do confirm that every patient did give written consent to participation.

Most IECs rely on regular, usually brief, written reports from investigators to reassure themselves that studies are being conducted in an ethical manner. They are implicitly assuming that doctors and other health professionals are fundamentally ethical individuals who will always put the best interests of participants in research first, and certainly before the needs of the research itself. Such an assumption may be less likely to hold when basic scientists with no background in the caring professions come to a decision that it is time to test their discovery on human subjects. In any case, events such as those involving paediatric cardiology in Bristol show that even highly qualified clinicians cannot always be relied upon to review and report on their performance systematically. ${ }^{23}$ The difficulties of ensuring that research is conducted in an ethical manner become far greater in multicentre studies that span major differences of culture. Relying on the "chaps" to "do the right thing" is really no guarantee that the interests of individuals participating in the research are being protected.

ACCESS TO MEDICAL RECORDS

Where clinical audit ends and clinical epidemiology begins is problematic. Both are inherently systematic inquiries and therefore fulfil part of the common definitions of research ${ }^{24}$ and they might be accommodated under the single, compound heading of "health services research". However, some IECs would only concern themselves with projects defined as "research" and would regard those defined as "audit" as being part of good clinical practice and therefore outside their brief. On the other hand, a strict view might be that using clinical files for any purpose other than that for which they were originally created, namely, to record the 
patient's condition and his or her response to treatment, should rightfully be subject to the approval of an ethics committee. The argument is not entirely academic as, increasingly, systematic review of individuals' practices is required as part of continuing accreditation and maintenance of professional standards. ${ }^{425}$

Use of identified clinical records in the training of health professionals is a related matter but one that seems to have escaped systematic consideration of the ethical questions it raises. Patients admitted to teaching hospitals are not always told that they may be asked to take part in an interview, examination or treatment conducted by a trainee professional, but their right to refuse to see or be seen by such individuals is widely acknowledged and respected. Even so, does any hospital require that students be excluded from meetings of clinical teams when the cases of patients who have declined to see students are discussed? If we have developed complex, albeit imperfect, systems and procedures for regulating research, why is there not the equivalent forma framework for regulating the clinical training of health professionals? Surely issues of confidentiality and autonomy and the risk of harm are more pressing when trainees rather than fully qualified practitioners are involved? The double standard that is very evident here only highlights the undue amounts of red tape in which we have now enmeshed research activity and underlines the need for a fundamental alteration in our approach.

\section{SELF EXPERIMENTATION}

There is a long tradition in medical research of experimenting on oneself. Some think that Hunter infected himself with syphilis, perhaps inadvertently, because his interest was in gonorrhoea. ${ }^{26}$ Banting may have tried a pancreatic extract on himself and certainly gave it orally to a diabetic colleague some months before it was first injected into a patient, ${ }^{7}$ and Marshall deliberately attempted to fulfil Koch's postulates with regard to the role of Helicobacter pylori in gastritis and peptic ulcer by swallowing a suspension of the organism. ${ }^{27}$ As the example of Hunter shows, self experimentation can be fatal, but should the charter of IECs explicitly include a brief to protect investigators from themselves as well as ones to safeguard the interests of other participants in research and of the institution in which the research is conducted?

INDIVIDUALS WHO ARE UNABLE TO GIVE CONSENT Apart from persons such as patients, prisoners and laboratory staff who may be in dependent relationships with research investigators, there are at least three other situations that raise difficult issues with regard to consent to participate in research. The first of these concerns retrospective studies of patients who have already died. On the one hand, there are jurisdictions where data protection laws prohibit access to routinely compiled death registrations by any non-governmental person. ${ }^{18}$ On the other hand, several countries have well established confidential inquiries into maternal ${ }^{28}$ and perinatal death ${ }^{29}$ that systematically review the records of such cases apparently without reference to any IEC. Indeed, in any population-wide review of medical care it is exactly the cases with an adverse outcome that may be of particular interest because of the likelihood that they might reveal sub-optimal management. ${ }^{4}$ It is clear that a blanket ban on such access may not be in the interests of the wider community, but the issues of the privacy of the deceased person and his or her family and of who might legitimately give consent to access to his or her records as part of the cycle of evidence-based practice, if an IEC insists that such consent is obtained, are not easily resolved.

A second general area of difficulty concerns research on individuals who may be incompetent to give consent by reason of age, cognitive impairment or mental illness. In practice, workable solutions to the issues raised by such research can usually be found, but of significance here is that there are no consistent rules. For example, otherwise competent children of sixteen are legally minors in many jurisdictions but their own consent, as well as that of a parent, would usually be sought before they took part in a research project. The absence of a single, generally accepted rule again underlines that current systems of ethical review still depend critically on the discretion and judgment of individual research investigators.

A final sometime area of difficulty concerns systematic trials of the management of medical emergencies. The easy argument is that if an investigator believes that a particular innovation is likely to represent a significant advance in the clinical management of such cases then there is an ethical duty to give that treatment to all such patients. This makes randomised controlled trials of emergency care effectively impossible, but the compelling example of thalidomide reminds us that a very important reason for conducting trials is to be sure that new methods are in fact not worse than established ones.

\section{PEOPLE OF DIFFERENCE}

"Modern medical science" is very much the medical science of Western Europe. Over the years investigators from this tradition have learnt a very great deal, first about the anatomy, then the physiology and biochemistry, and now the genetics of certain small ethnic groups in the Arctic, North America, the Kalahari and Australia. Studies of closed communities living in harsh environments have revealed much about the capacity of the human species for physical and social adaptation. In general, however, the subjects of these investigations have gained little from this intensive research effort and now increasingly wish to exercise some degree of control over all such activities involving their people. Designing and conducting studies in ways that are acceptable to communities that are "different" is a complex challenge. The list of what may be relevant "differences" is a long one, covering at least notions of creation and causality, private and public, secret and secular, the person and the polity. This makes it entirely foolish to 
attempt to write an all-encompassing set of rules or guidelines. Success depends on the investigator having, and sensitively applying, knowledge of the relevant cultural terrain. An ethics committee may be able to identify and counsel the uninformed or misguided novice, but the project stands or falls on the attributes of the investigator, not those of the committee.

\section{GENETIC RESEARCH}

In a sense, among the smallest "different" groups of all are the sets of the individuals carrying certain genetic markers or mutations. As a community, we have not begun to consider seriously and carefully the personal or social consequences of being able to assign, even in utero, relatively precise lifetime probabilities for this or that individual developing a certain physical or mental condition. The problem for ethics committees is that they are faced with these difficult questions now, and that in contrast with well established notions of, for example, individual privacy and autonomy, the whole field of genetic research is so new and is moving so fast that guiding landmarks are very few.

There is not even broad agreement on the answers to two questions that recur repeatedly in this area of research. These are, firstly, whether an individual who provides genetic material to an investigator can give valid "blanket" consent in advance for that material to be tested for any genetic marker, some markers and tests themselves having not yet been described, and, secondly, what are the duties of the investigator to the donor of that material (and perhaps the family of the donor) when the laboratory happens upon a finding of potential personal significance. Thus, current systems of ethical oversight are lagging well behind the capabilities of the science and frequently it is left to individual investigators to set and follow rules for their work.

\section{Summary of the problem}

It is clear that systems of ethical oversight designed primarily to regulate intervention studies involving individual patients associated with single institutions have been completely overtaken by developments in clinical, molecular and epidemiological research. There is also abundant evidence that many of the present systems struggle to accommodate multicentre or population-wide investigations and fail to appreciate the differences in practical and ethical issues associated with observational as opposed to experimental studies. At the same time, too much emphasis is placed upon initial approval of study protocols and too little regard is given to whether the investigators are competent to do the work and whether the work is conducted in an ethically appropriate manner. In effect, then, most systems are relying on unchecked assumptions as to the good judgment and integrity of individual investigators.

At best current arrangements impede important research while providing little guarantee that the research that is done is conducted according to accepted standards of ethical practice. In the worst cases, the priority given to concerns for the confidentiality of individuals over the desire of the community for confidence in the safety and efficiency of their health system is so great that certain types of research enquiry cannot be conducted at all. Further, current arrangements omit to provide for several other types of non-clinical activities involving human subjects. Finally, the scale and speed of developments in human genetics suggest that even if we could correct the obvious deficiencies in our present arrangements for ethical review, we are unlikely ever to be able fully to anticipate advances in medical science or prepare adequate systems for its regulation.

\section{Where do we go from here?}

In addressing the present situation, our choices appear to lie between continuing to make sequential and piecemeal amendments to current arrangements or undertaking a far-reaching overhaul. To pursue the first of these options is to persist with a policy that has contributed directly to our present difficulties. The alternative, defining again the goals that we are trying to achieve and designing a new system with these in mind, therefore bears serious consideration.

In addition to such time-honoured principles as primum non nocere and providing reasonable (not necessarily absolute) protection of the rights of individuals to autonomy and privacy, a new system should explicitly take into account the need of the community to have confidence in the safety, efficacy and efficiency of its health system, and in the safety of other areas of life, such as domestic, public and occupational environments. It should also make it clear that any system ultimately depends on the behaviour of individual investigators.

Acceptance of this framework would lead to three important changes in our systems of ethical oversight. First, there would be a much stronger emphasis on education and debate about ethical questions, both among health professionals and others conducting research involving human subjects and in the general community. Too often, if they arise at all, ethical issues come as an afterthought in planning and conducting research, whereas increasing the general level of attention given to ethical matters should mean that these questions are considered early and often in individual investigations.

Secondly, we will move to a system akin to licensing for drivers (and medical practitioners), one in which a period of formal training in research, which will cover the ethical questions related to research on human subjects and material obtained from them, will be followed by a qualifying examination, a period of probationary practice under supervision, and then, subject to satisfactory performance, a full licence. Normally this licence would be automatically renewed, but if certain (combinations of) offences were detected it would be suspended or cancelled. In practice there would 
be a requirement that fully licensed investigators keep relevant ethics committees informed as to their research activities, with strict sanctions applying in cases of failure to fulfil this obligation. With regard to responsibilities for disclosure and for exercise of discretion and judgment, these arrangements differ little in essence from those currently in place. Indeed, they would have the virtue of making formal fundamental but largely unacknowledged features of many current systems.

Thirdly, ethics committees would devote much less of their time and energy to scrutinising applications to conduct medical, epidemiological and other research involving human subjects, and much more time to monitoring research activity in efforts to detect unlicensed drivers and those infringing the rules of the research road.

\section{Acknowledgements}

I am grateful to Irene Stratton for providing helpful comments on an early draft of this paper.

Konrad famrozik, MBBS, DPhil, FAFPHM, is Professor of Public Health, University of Western Australia.

\section{References}

1 United Kingdom. Animals (Scientific Procedures) Act, 1986.

2 National Health and Medical Research Council. National statement on ethical conduct of research involving humans. Canberra: NHMRC, 1999.

3 Office of Inspector General. Institutional Review Boards: their role in reviewing approved research. Washington: US Departmen of Health and Human Services, Office of Inspector General, Office of Evaluation and Inspections, 1994.

4 Department of Health. Clinical governance in the new NHS. London: Department of Health, 1999 (health service circular 1999/065)

5 Yusuf S, Collins R, Peto R. Why do we need some large, simple randomized trials? Statistics in Medicine 1984;3:409-40.

6 Kasten FH. Paul Ehrlich: pathfinder in cell biology. 1. Chronicle of his life and accomplishments in epidemiology, cancer research, and chemotherapy. Biotechnic and Histochemistry 996;71:2-37

7 Bliss M. The discovery of insulin. Toronto: McClelland and Stewart, 1982

8 Shuster E. The Nuremberg Code: Hippocratic ethics and human rights. Lancet 1998;351:974-7.
9 World Medical Association. Declaration of Helsinki: recommendations guiding physicians in biomedical research in human subjects. Helsinki: World Medical Association, 1964 (amended Tokyo 1975, Venice 1983).

10 Medical Research Council. Streptomycin treatment of pulmonary tuberculosis. British Medical fournal 1948;ii:769-82.

11 ISIS-4 Collaborators. ISIS-4: a randomised factorial tria assessing early oral captopril, oral mononitrate, and intravenous magnesium sulphate in 58,050 patients with suspected acute myocardial infarction. Lancet 1995;345:669-85.

12 Jamrozik K, Kolybaba M. Are ethics committees retarding the improvement of healthcare services in Australia? Medical fournal of Australia 1999;170:26-8.

13 Stevenson MR, Jamrozik K, Spittle J. A case-control study of Stevenson MR, Jamrozik K, Spittle J. A case-control study of
traffic risk factors and child pedestrian injury. International traffic risk factors and child pedestrian
fournal of Epidemiology 1995;24:957-64.

14 Shurtleff D, Kannel WB, Gordon T. The Framingham Study: an epidemiological investigation of cardiovascular disease. Washington: US Government Printing Office, 1970

15 Wynder EL, Graham EA. Tobacco smoking as a possible etioogic factor in bronchogenic carcinoma. Fournal of the American Medical Association 1950;143:329-36.

16 Doll R, Hill AB. Smoking and carcinoma of the lung. British Medical fournal 1950;ii:739-48.

17 Jamrozik K. Ethics committees and bureaucracy. Medical fournal of Australia 1997;167:51-4.

18 Bracken MB. Alarums false, alarums real: challenges and threats to the future of epidemiology. Annals of Epidemiology 1998;8:79-82.

19 White AE. Research ethics committees at work: the experience of one multi-location study. Fournal Medical Ethics 1996;22:352-5.

20 Ahmed AH, Nicholson KG. Delays and diversity in the practice of local research ethics committees. Fournal of Medical Ethics 1996;22:263-6.

21 Beran RG. Should there be an accredited ethics committee system for centralised review of multicentre clinical research? Medical fournal of Australia 1998;168:174-6.

22 Smith MA, Jalaludin B, Leeder SR, Smith WT. Isn't one institutional ethics committee's approval enough? Medical fournal of Australia 1994;160:662.

23 Dyer C. Bristol doctors found guilty of serious professional misconduct. British Medical fournal 1998;316:1924.

24 Pearsall J, Trumble B, eds. The Oxford English reference dictionary. Oxford: OUP, 1995.

25 Faculty of Public Health Medicine. Maintenance of professional standards. Sydney: Royal Australasian College of Physicians, 1998.

26 Wright DJ. John Hunter and venereal disease. Annals of the Royal College of Surgeons of England 1981;63:198-202.

27 Marshall BJ, Armstrong JA, McGechie DB, Glancy RJ. Attempt to fulfil Koch's postulates for pyloric campylobacter. Medical fournal of Australia 1985;142:436-9.

28 Department of Health. Why some babies die: report on confidential enquiries into maternal deaths in the United Kingdom, 1994-1996. London: The Stationery Office, 1998.

29 Thornton CM, O'Hara MD. A regional audit of perinatal and infant autopsies in Northern Ireland. British fournal of Obstetrics and Gynaecology 1998;105:18-23. 